\title{
Facial numbness in the ophthalmology clinic. A portentous sign
}

\author{
Shoaib Ugradar ${ }^{1} \cdot$ Laura Bonelli $^{1}$ Daniel Rootman ${ }^{1,2}$
}

Received: 1 April 2019 / Revised: 25 June 2019 / Accepted: 16 July 2019 / Published online: 30 August 2019

(c) The Author(s), under exclusive licence to The Royal College of Ophthalmologists 2019

\begin{abstract}
Aim To report a case series of 14 patients presenting with facial numbness primarily to the ophthalmology clinic.

Methods All patients presenting with facial numbness to the ophthalmology clinic at the University of California, Los Angeles, were reviewed for study entry between 1993 and the present. Patients with a history of trauma or surgery were excluded.

Results Fourteen patients (eight females and six males) presented to the ophthalmology clinic with numbness. Nine patients (64\%) presented primarily with numbness. This symptom was associated with mortality (57\%) and significant morbidity. The most common cause was neoplastic pathology $(n=10,71 \%)$, with perineural spread from squamous cell carcinoma being the most common (five cases, 36\%). The remaining cases were related to infection $(n=4,29 \%)$.

Conclusions Most of the patients presenting to our service with numbness eventually died due to their condition. Given the poor prognosis of the patients in our case series, numbness of the face may be a portentous sign and therefore warrants a thorough examination with close follow up.
\end{abstract}

\section{Introduction}

The trigeminal nerve provides sensory input from the face, scalp, cornea and mucosal linings of the mouth and nasal cavity [1]. The nerve is susceptible to injury and disease at any point from its origin at the pons throughout its course along the skull base to the superficial facial structures that it supplies. Pain due to trigeminal neuralgia is an early feature of dysfunction and usually prompts patients to seek urgent medical attention. However, trigeminal neuropathy (TNO) characterized by numbness can be more indolent, often going unnoticed by the patient.

The nerve may be affected by a spectrum of pathological processes ranging from vascular lesions and inflammatory insults to traumatic and neoplastic disorders. Although epidemiological data regarding incidence and prevalence are scarcely available, the most common causes of TNO

Shoaib Ugradar

ugradsahi@aol.com

1 The Jules Stein Eye Institute, University of California, Los Angeles, CA, USA

2 The Doheny Eye Institute, University of California, Los Angeles, CA, USA tend to be iatrogenic, traumatic, cerebellopontine angle (CPA) tumours and idiopathic neuropathy $[2,3]$.

Due to the intimate association of the nerve and its branches to other structures around the eye and orbit, patients with TNO may initially present with numbness to an ophthalmologist. This study describes a series of patients presenting to the ophthalmologist's office with facial numbness, emphasizing the serious and often fatal underlying diagnoses for this disorder.

\section{Anatomy of the trigeminal nerve}

The trigeminal nerve is the largest cranial nerve, consisting of a sensory and motor root. The sensory root arises from the lateral surface of the pons passing forwards within the pia-arachnoid space in the posterior fossa. At this point, it passes lateral to the abducens nerve, through a groove on the medial aspect of the upper petrous temporal bone. As it continues forward, it pierces the dura to supply the Gasserian ganglion, crossing over the facial and auditory nerves as they enter the internal auditory foramen [4].

The Gasserian ganglion lies within a dural envelope in a depression named Meckel's cave, near the tip of the petrous part of the temporal bone that is posterior, lateral and inferior to the cavernous sinus. The internal carotid artery passes laterally to the ganglion supplying a few sympathetic 
fibres form its plexus. The three major divisions of the trigeminal nerve, the ophthalmic (V1), maxillary (V2) and mandibular nerves (V3), arise from the anterior portion of the Gasserian ganglion [1].

\section{The ophthalmic division (V1)}

The ophthalmic division is the smallest of the three branches. It passes along the lateral wall of the cavernous sinus under the trochlea nerve and above the maxillary division (V2). It enters the superior orbital fissure and divides into three branches: frontal, lacrimal and nasociliary. The frontal branch is the largest of the three and passes along the roof of the orbit beneath the periosteum and divides further into the supraorbital and supratrochlear nerves. The supraorbital portion exits the orbit via the supraorbital foramen and supplies the forehead, scalp, upper eyelid and underlying conjunctiva. The supratrochlear nerve exits the orbit in the region of the trochlea and supplies the medial part of the forehead and upper eyelid along with the base of the nose. The superior division of the lacrimal nerve supplies the gland and the skin of the lateral upper and lower lids. The nasociliary nerve receives sensory input from the eye and enters the orbit through the annulus of Zinn along with the ophthalmic artery, running beneath the superior rectus muscle towards the medial wall. Branches to the ethmoidal neurovascular bundles exit the orbit to receive innervation from the nasal cavity and the skin of the middle and lower part of the nose [4].

\section{The maxillary division (V2)}

After leaving the anterior portion of the Gasserian ganglion, it passes within the lateral wall of the cavernous sinus, clustered with V1 and the other cranial nerves in the posterior cavernous sinus and separating inferiorly as it passes anteriorly to the foramen rotundum. It then crosses the pterygopalatine fossa to enter the orbit through the inferior orbital fissure. In the orbit, it is known as the infraorbital nerve and runs within the inferior orbital canal to exit the orbit via the infraorbital foramen. Here, it divides into the inferior palpebral, lateral nasal and superior labial branches, supplying the corresponding regions of the face [4].

\section{The mandibular division (V3)}

The mandibular division also arises from the anterior portion of the Gasserian ganglion, coursing downward through the foramen ovale to enter the nasopharyngeal masticator space, where it provides motor supply to the muscles of mastication and sensation to the lower lip, chin, tongue and mandibular teeth [1].

\section{Patients and methods}

In this consecutive case series, patients presenting with numbness to the ophthalmology service at the Jules Stein Eye Institute, UCLA, were reviewed. Patients were identified from an institutional database compiled from 1993 to the present. The institutional review board at the University of California, Los Angeles, provided ethics approval for the study.

Patients with a history of trauma and surgery were excluded. Records were evaluated for race, age at presentation, laterality, other clinical features as well as anatomical location of the lesion, surgery and final diagnosis. Patient outcome was assessed through association with other services. Data are presented in observational format.

\section{Group characteristics}

Fourteen patients presented to the ophthalmology service with numbness of the face during the study period. The mean (SD) age at presentation was 67.1 (16.17). There was a slight female preponderance (57\%) (Table 1). Sixty four percent of the patients were Caucasian.

Five cases (36\%) were diagnosed with squamous cell carcinoma and three patients $(21 \%)$ presented with fungal invasion of the orbital apex and cavernous sinus. The remaining cases included melanoma (1), metastatic adenocarcinoma (1), nasopharyngeal carcinoma (1), cavernous schwannoma (1), carcinomatous meningitis (1) and bacterial cellulitis (1). Nine cases (64\%) had V1 involvement, while 10 cases $(71 \%)$ included V2 involvement and 4 cases (29\%) demonstrated involvement of V3.

Thickening of the branches of the trigeminal nerve was seen on MRI of three cases, (cases 9, 12 and 13), while abnormal enhancement was seen in Meckel's cave in two cases (cases 1 and 2). Non-specific orbital inflammation was seen in the four cases involving infection (three fungal and one bacterial). A histological diagnosis was made in the ten patients with a solid tumour and one case of fungal cavernous sinus disease following biopsy.

Following diagnosis, eight patients were placed on palliative care, while three patients were offered chemotherapy and radiation. Three patients were treated with intravenous antifungals and one patient received intravenous antibiotics. One of the patients (case 9) had transsphenoidal skull base surgery to debulk the tumour.

At most recent follow up (mean 12.5 mo, SD 10.7), eight patients $(57 \%)$ had perished due to the diagnosis responsible for their initial presentation, three patients $(21 \%)$ were currently on chemotherapy for the disease process and three patients (21\%) survived the pathologic process and were currently not being treated actively. These patients were all 


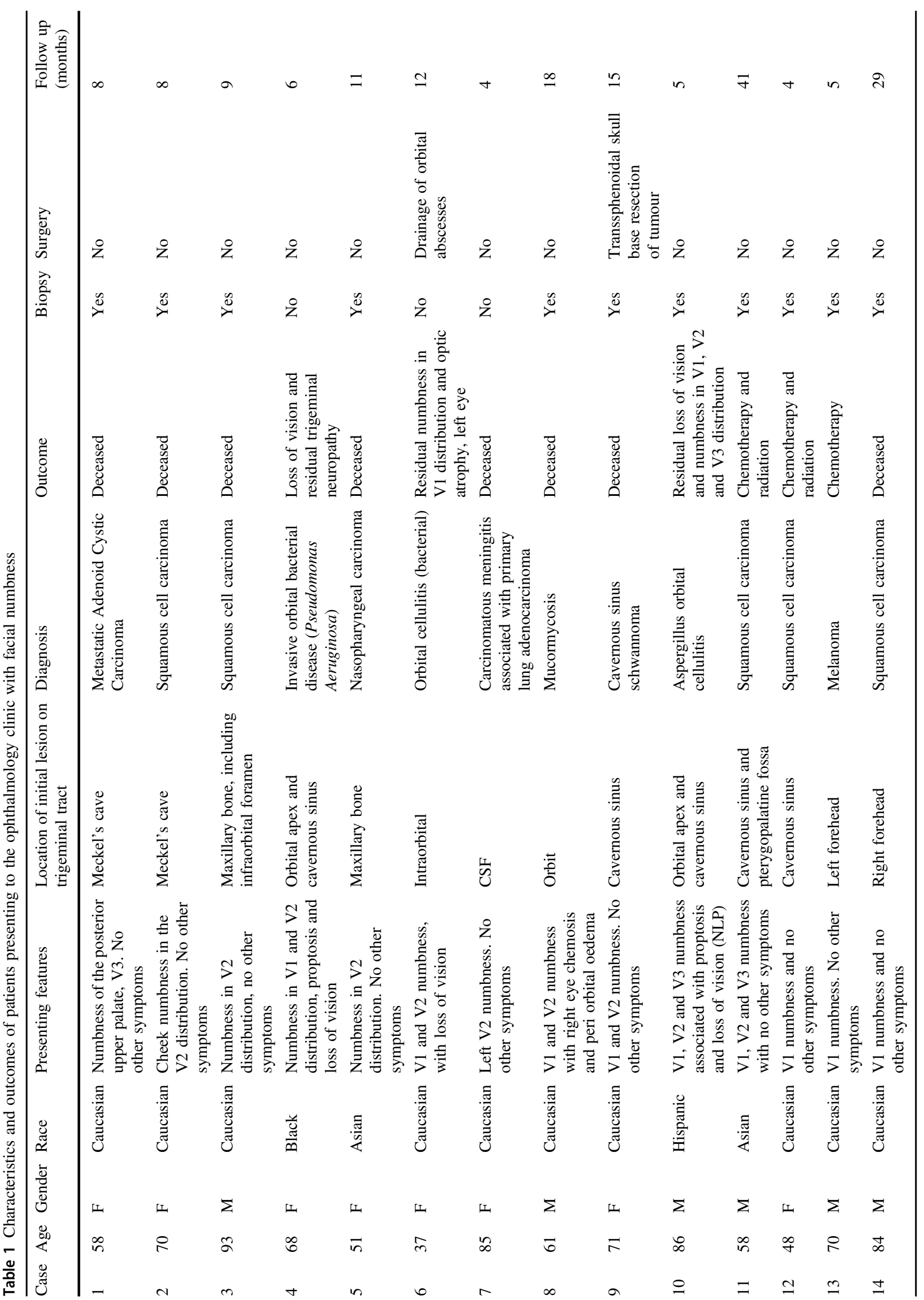


left with residual dysfunction of the affected trigeminal branches. No patients had signs of trigeminal neuralgia at follow up. Two representative cases are presented.

\section{Case 7}

An 85-year-old female presented to the ophthalmology service with a 1-month history of numbness on the left side of her face. She did not endorse any other symptoms at this time. On examination, visual function and ocular examination were both normal. She did not have any systemic symptoms or examination findings. A contrast MRI scan did not reveal any abnormalities.

Two weeks after her initial presentation, the patient developed vertical diplopia and signs of right facial paralysis in the lower motoneuron distribution. A lumbar puncture did not reveal signs of infection but did find cells positive for keratin $\mathrm{AE} 1 / 3$, suggestive of a malignant pathology. A subsequent CT scan of the chest revealed a lesion at the apex of the left lung (Fig. 1). Lung biopsy was performed and a diagnosis of carcinomatous meningitis secondary to lung adenocarcinoma was made. The patient succumbed to pneumonia 3 weeks after initial presentation.

\section{Case 12}

A 48-year-old female presented with a 4-month history of numbness on the left upper forehead and an aching pain around the orbit. On examination, numbness was noted in the V1 distribution. Visual function was normal. There were no abnormalities on intraocular, orbital or facial examination and a full systemic assessment was non-contributory. No history of skin or other cancer was noted.

Magnetic resonance imaging revealed abnormal enhancement of the left cavernous sinus, frontal nerve (Fig. 2), lacrimal gland, superior rectus and superior orbital

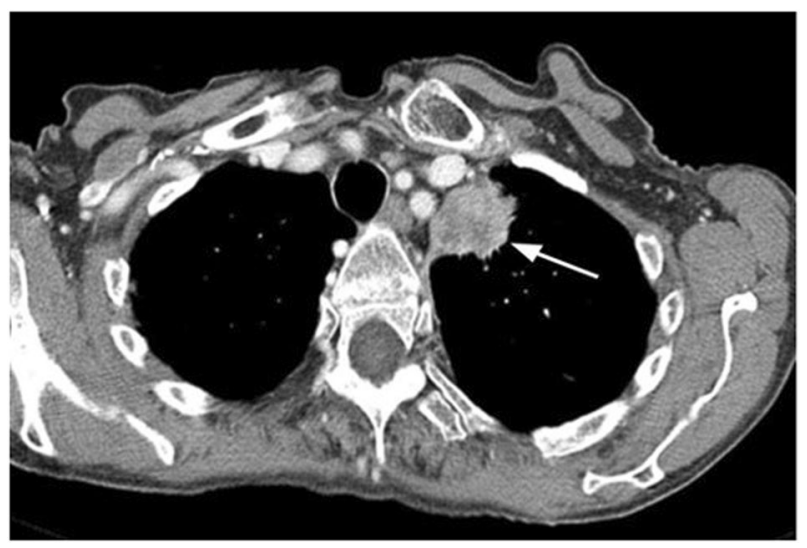

Fig. 1 CT scan of the chest revealing a lesion in the apex of the lung fissure. The patient was initially diagnosed with Tolosa Hunt syndrome and commenced steroid therapy. She did not respond to therapy and a biopsy was performed involving tissue samples from the left lacrimal gland and frontal nerve. The frontal nerve biopsy revealed squamous cell carcinoma with perineural spread, the lacrimal gland demonstrated non-specific inflammation. As a result of this diagnosis, chemotherapy with cisplatin and radiotherapy was initiated.

\section{Discussion}

In this case series, numbness was the presenting feature in nine patients who attended the ophthalmology department over a 25 -year period. This symptom, although rare, was associated with a mortality of $57 \%$. The most common cause was neoplastic pathology $(n=10,71 \%)$, with perineural spread from squamous cell carcinoma being the most common among these (five cases, 36\%). The remaining cases were related to infection $(n=4,29 \%)$. Of the six patients that survived, three $(21 \%)$ had associated loss of vision, while six $(100 \%)$ had residual numbness.

Tumours may affect the trigeminal nerve at various locations along its course in the intracranial space from the brainstem at the CPA to the Gasserian ganglion and through the cavernous sinus. Due to proximity of other cranial nerve nuclei in the brainstem, isolated nuclear TNO is uncommon.

The CPA is an area where the trigeminal nerve is closely related to CNs VII and VIII. Typical lesions at this level

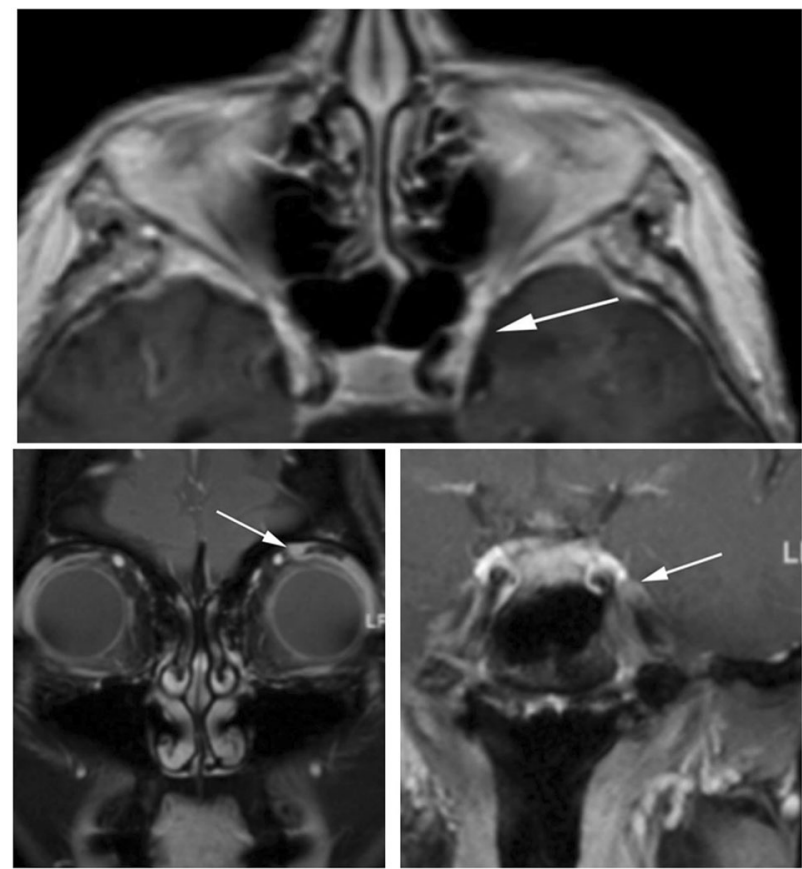

Fig. 2 MRI showing areas of perineural thickening (white arrows) 
include trigeminal schwannomas, meningiomas and epidermoid cysts [1]. A review of 40 patients with CPA meningiomas found that $16.2 \%$ of patients had TNO on presentation [5]. In our series, we did not see any patients with lesions in the CPA. This may be a result of referral bias as patients with CNs VII and VIII related symptoms may have presented to other services.

The Gasserian ganglion is found within Meckel's cave, a dura-covered impression of the petrous portion of the temporal bone. This region is commonly affected by trigeminal schwannomas, meningiomas and metastatic lesions from perineural spread. In our series, two patients presented with lesions in Meckel's cave. The first (case 1) occurred due to metastatic spread of adenoid cystic carcinoma, while the second (case 2) was due to perineural spread of a squamous cell carcinoma.

The second and third divisions of the trigeminal nerve (V2 and V3) are most commonly affected by perineural spread [6,7]. Numb chin syndrome [8] describes a clinical entity where patients present with numbness of the chin and lower lip in relation to lesions affecting branches of the mandibular branch of the Trigeminal nerve (V3). This condition is worrisome since it can frequently be associated with malignancy [9]. Retrograde perineural spread from any of the sites innervated by V2 can occur along the distal branches, often from lesions involving the pterygopalatine fossa. Tumours around the maxillary sinus, for instance a primary nasopharyngeal cancer (case 5), may also spread perineurally along V2.

Lesions of the cavernous sinus were found in three patients (21\%). Two cases were due to extension of squamous cell carcinoma, while one was secondary to fungal disease in the cavernous sinus and the orbital apex.

In our study, isolated TNO was rare and associated with serious pathology. It appears as though trigeminal hypesthesia is less common than other motor nerve palsies in the region. It is not completely clear why this is the case. Initially, it could be simply related to selection bias, as more chronic and systemic disease-related trigeminal dysfunction may have presented to other services [1]. However, this does not fully explain the severity of disease we described. We did not find any cases of isolated 'ischaemic' trigeminal palsy nor that trigeminal palsy was a significant feature of Tolosa Hunt syndrome. This is despite the relative preponderance of IV, VI and III nerve palsy by these mechanisms [10]. It is possible that the structure and organization of the nerve might protect it from vascular pathology. The sensory fibres of the trigeminal nerve that are responsible for transmitting pain and touch are unmyelinated. These fibres are more resistant to injury and are more easily regenerated than their myelinated counterparts [11]. Therefore, following non traumatic injury, unmyelinated fibres recover in greater number, while the myelinated fibres degenerate [11]. This may mean that the trigeminal nerve, due to its larger size and unmyelinated fibres, may be more robust and perhaps carry greater redundancy, and only be clinically affected when serious pathology is present. In addition, the position of the nerve within a dural envelope within both Meckel's cave and the cavernous sinus may offer a relative insulation from inflammatory injury.

It is also noteworthy that $8 / 14$ cases we reported were related to perineural spread of a sinus or skin tumour. The dermatomal organization of small end fibres may be responsible for the greater incidence of perineural spread we noted. The wide distribution of sensory fibres thus increases the likelihood of contact with a tumour that has trophic bias towards perineural spread. Motor neurons being less widely distributed would be less commonly encountered targets. Of course, it is alternatively possible that there is an unknown specific biochemical attraction for certain tumours to sensory nerves, leading to such a sensory bias in perineural invasion. Overall, there are a wide variety of potential explanations for the rarity and severity of trigeminal hypesthesia, and it is most likely that one mechanism does not explain all cases. Further research will be required to understand these pathophysiologic relationships.

Although TNO may be caused by benign conditions [3], our case series highlights that patients presenting to an ophthalmologist may have a worse prognosis. Most of the patients presenting to our service with numbness eventually died because of their condition, while all remaining survivors were affected by conditions associated with significant morbidity. Given the poor prognosis of the patients in our case series, numbness of the face may be a portentous sign and therefore warrants a thorough examination with close follow up.

\section{Summary}

\section{What was known before}

- It was uncertain how important facial numbness in the ophthalmology clinic may be.

\section{What this study adds}

- This study shows that over $50 \%$ of patients who presented with facial numbness died.

\section{Compliance with ethical standards}

Conflict of interest The authors declare that they have no conflict of interest. 
Publisher's note: Springer Nature remains neutral with regard to jurisdictional claims in published maps and institutional affiliations.

\section{References}

1. Smith JH, Cutrer FM. Numbness matters: a clinical review of trigeminal neuropathy. Cephalalgia. 2011;31:1131-44. http:// journals.sagepub.com/doi/10.1177/0333102411411203. Acessed 19 Feb 2019.

2. Goldstein NP, Gibilisco JA, Rushton JG. Trigeminal neuropathy and neuritis: a study of etiology with emphasis on dental causes. JAMA J Am Med Assoc. 1963;184:458-62.

3. Horowitz SH. Isolated facial numbness. Clinical significance and relation to trigeminal neuropathy. Ann Intern Med. 1974;80:49-53.

4. Newell FW. Sir Stewart Duke-Elder-the system of ophthalmology. Am J Ophthalmol. 1977;83:594-9.

5. Voss NF, Vrionis FD, Heilman CB, Robertson JH. Meningiomas of the cerebellopontine angle. Surg Neurol. 2000;53:439-46. http://www.ncbi.nlm.nih.gov/pubmed/10874142. Accessed 21 Feb 2019.
6. Ballantyne AJ, Mccarten AB, Ibanez ML. The extension of cancer of the head and neck through peripheral nerves. Am J Surg. 1963;106:651-67. http://www.ncbi.nlm.nih.gov/pubmed/ 14070757. Accessed 21 Feb 2019.

7. Parker GD, Harnsberger FHR. Clinical-radiologic issues in perineural tumor spread of malignant diseases of the extracranial head and neck. https://pubs.rsna.org/doi/pdf/10.1148/radiographics.11. 3.1852933. Accessed 21 Feb 2019.

8. Smith RM, Hassan A, Robertson CE. Numb chin syndrome. Curr Pain Headache Rep. 2015;19:44. http://link.springer.com/10. 1007/s11916-015-0515-y. Accessed 25 Jun 2019.

9. Galán Gil S, Peñarrocha Diago M, Peñarrocha Diago M. Malignant mental nerve neuropathy: systematic review. Med Oral Patol Oral Cir Bucal. 2008;13:E616-21. http://www.ncbi.nlm.nih.gov/ pubmed/18830167. Accessed 25 Jun 2019.

10. Tamhankar MA, Volpe NJ. Management of acute cranial nerve 3, 4 and 6 palsies. Curr Opin Ophthalmol. 2015;26:464-8. http://www.ncbi.nlm.nih.gov/pubmed/26367093. Accessed 4 Mar 2019.

11. Jones R. Repair of the trigeminal nerve: a review. Aust Dent J. 2010;55:112-9. http://doi.wiley.com/10.1111/j.1834-7819.2010. 01216.x. Accessed 26 Feb 2019. 